\title{
Le blason du calice sur les apis Mameloukes
}

\author{
Radwa M. Omar \\ Lecturer of Islamic Architecture, Faculty of Tourism and Hotels Management, Suez Canal University
}

\section{Résumé}

Les blasons chez les musulmans, leurs formes, leur origine et leur fonction, est un sujet qui a fait couler beaucoup d'encre. Il existait diverses sortes de blasons, personnel ou fonctionnel, simples ou composés, temporels ou permanents. Cette recherche traite un blason fonctionnel d'une importance primordiale, et qui est figuré sur divers monuments et pièces d'arts appliqués, c'est le blason du calice, attribué aux échansons; une charge assez sensible dans la cour du sultan et dont le responsable devait, à l'époque, jouir d'une confiance ultime de la part du sultan pour la remplir. Parmi les divers arts appliqués, cette recherche met l'accent sur le blason du calice, exécuté sur les tapis mamelouks, sans doute, considérés comme des pièces artistiques distinctes dont les techniques de la fabrication ainsi que les couleurs lui offrent une identité unique. Les musées d'arts à l'étranger comportent des chefs- d'œuvres des tapis fabriqués en Egypte sous les mamelouks, la présente contribution en analyse quelques pièces, comportant le blason d'échanson sous différentes formes et couleurs.

Mots -clés: Tapis, Mamelouke, blason, calice, échanson, arts appliqués.

\section{Les Origines du blason dans l'Art Musulman}

Aussi loin dans l'histoire, le symbolisme jouait un rôle assez frappant dans les légendes des peuples, ayant une valeur religieuse, magique ou astrologique, il représentait un besoin inné de l'esprit humain comme la parole et l'écriture. ${ }^{1}$

Après la conquête Arabe, qui monopolisait, en moins de cent ans, la Syrie, la Mésopotamie, l'Egypte, la Perse, l'Espagne, des parties de la France et de l'Italie ainsi que les îles de la Méditerranée, les infiltrations scientifiques, culturelles, artistiques et même coutumières, étaient continuelles entre les pays occidentaux et orientaux. Une des sciences qui était le plus en honneur est l'astrologie que les arabes étudiaient avec passion malgré une certaine interdiction dans leur religion qui les prohibait de rechercher le futur, pourtant, les maîtres de cette science étaient des grecs et des perses qui ont traduit leurs œuvres, essentiellement emblématiques, en arabe; et c'est ainsi que les emblèmes héraldiques, perse et grecque se sont infiltrés dans l'esprit, la culture et les coutumes arabes. ${ }^{2}$

Selon Yacoub Artin Pasha, la première connaissance au blason était faite par «Al- Firdawsī» dans son ouvrage épique «le Livre des Rois», non seulement l'auteur a fait réveiller le souvenir des ancêtres et de leurs gloires nationales, mais aussi, il a incité à l'étude de l'astrologie qui était déjà répandue et a créé le goût du symbolisme graphique, c'est alors, «al- Firdawsī» qui a mentionné, dans ses vers, des symboles personnels et des couleurs qui formaient de véritables armoiries, blasonnant les rois, les héros et les guerriers. Ces armoiries les font reconnaitre dans les tournois, les fêtes et même sur les champs de bataille.

Ainsi, les sultans et leurs Mameloukes, en lisant le «Šāhnāmeh», ont beaucoup apprécié les coutumes des rois et des héros glorifiés dans cet œuvre, par suite, ils commençaient à adopter leurs armoiries avec ses couleurs et des symboles graphiques. ${ }^{3}$

\section{L'étymologie du mot 'Rank'}

Blason, écusson ou armoirie ${ }^{4}$, sont des termes qui signifient 'rank'; le pluriel est 'runūk', c'est un mot persan qui veut dire 'couleur', il a été particulièrement appliqué aux couleurs de la bannière et de là à celles de l'écusson ou des armoiries, et qui a pris par la suite la signification de symbole indiquant le rang ou la fonction du chevalier qui le portait. $^{5}$

D'habitude, à l'occasion de célébrer l'émirat d'un Mamelouke, on lui attribue un blason «rank», qui était une sorte d'armoirie de dignité, ou un emblème privé comme la rosace de lis qu'a choisi «Nūr ad- Dīn Mahmmūd Zankī», et la fleur à six pétales choisie par la famille «Qālāwūn». Un autre exemple est fourni par le sultan «Az- Zāhir Baybars al- Bunduqdārī» et son fils «As- Sa ${ }^{\top} \overline{1}$ B Baraka Hāan» qui ont choisi le lion. ${ }^{6}$

Un autre genre de blason était le blason fonctionnel, qui indique la profession dans la cour du sultan, comme l'encrier, emblème du Dawādār ${ }^{7}$, l'épée du silaḥdār ${ }^{8}$, le fer à cheval de l'émir Ahūro ${ }^{9}$ et la coupe, blason de l'échanson ${ }^{10}$. 
Ces emblèmes indiquaient des charges de cour, mais important à noter que le titulaire ne prenait des armoiries rappelant ses anciennes charges de cour qu'au moment où il était nommé émir.

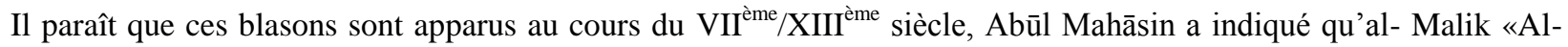
Șāli ḥ Nağm al- Dīn Ayyūb» a choisi Aybak pour remplir la fonction de dégustateur, «et, pour ce motif, il lui a donné comme armoiries, lorsqu'il l'élevé à la dignité d'émir, l'image d'une petite table».

$$
\text { "ولهذا لما أمره كان عمل رنكه صورة خونجا". } 11
$$

A certaines occasions, ces blasons ont été attribués à des mamelouks à titre temporaire, comme c'était le cas de Yašbak ${ }^{12}$ qui a été blasonné d'un lion par le sultan Qāytbāy lors de son commandement d'un corps d'armée, probablement, cette armoirie était simplement pour la durée de l'expédition prévue. ${ }^{13}$

Au début, les blasons figuraient d'une façon simple, sans contour, plus tard, ils ont été dessinés dans des cercles ou des formes ovales, bipartites ou tripartites où les symboles appariassent dans les deux ou trois registres qui les composent. ${ }^{14}$

Encore, ces blasons ont été colorés par des couleurs distinctes, choisies par l'émir qui les portaient, toujours selon des règles strictes à peur qu'ils soient confondus. ${ }^{15}$

Il existait des blasons simples et d'autres composés, il est vital de noter que l'existence de ces formes était simultanée, et parfois, ils représentaient une évolution chronologique. Ces blasons composés ont fait leur apparition sous le règne d'al- Zāhir Baybars al- Bunduqdārīî', propagés sous le règne d' 'al- Nāṣir Muhammad ibn Qalāwūn'17, au début, il contenait l'emblème du sultan et le blason indiquant la charge de l'émir dans la cour du sultan, puis, sa forme s'est réduite au blason personnel actuel en addition des blasons référant aux anciens postes remplis par cet émir. ${ }^{18}$

\section{L'importance du poste d'échanson 'Sāqī'}

L'échanson du sultan était un des personnages les plus importants de la cour, il était responsable de présenter les boissons au sultan, une charge sensible à l'époque, ce qui explique qu'il était le compagnon intime du souverain, et évidemment, il jouissait de toute sa confiance. Une série considérable d'officiers mamelouks ont rempli ce poste, les échansons et les secrétaires ont fourni les futurs commandants de mille, et à tour de rôle, ils ont reçu un blason fonctionnel - le calice- indiquant leur charge. ${ }^{19}$

Ce poste était parfois héréditaire, un émir pouvait recevoir le blason de la coupe de son père, même s'il ne remplissait pas la fonction d'échanson comme c'était le cas de «Aḥmad ibn Buktumr» et «Ḥusayn ibn Qawșūn». ${ }^{20}$

Parmi les émirs mamelouks qui ont rempli la charge d'échanson: 'Kitbūg̀ā al- Manșūrī', devenu sultan en l'an (694 H. /1294 J.C), 'Ṭunbuġā al- Maridān̄̄ ${ }^{21}$, le sultan 'al- Mu' ayyad Šayh ${ }^{22}$, le sultan 'Barqūq' et 'al- Ašraf Ğānblāṭ'. ${ }^{23}$

\section{Le blason du calice}

L'emblème de la coupe était le plus fréquemment employé sur les monuments islamiques ainsi que sur les arts appliqués. Cette expansion d'usage est très probablement due, d'une part, au grand nombre des émirs qui ont subi le titre d'échanson, surtout ceux qui étaient proches du sultan, d'autre part, à leur supériorité sur d'autres postes sultaniens. ${ }^{24}$

Les monuments et les arts appliqués nous ont fourni de diverses formes du blason de la coupe, parfois simple, sans contour, et d'autre fois, dans un cercle, où la coupe remplissait presque la totalité de son aire, ou occupant les deux tiers du blason bipartite ou une des bandeaux formant le blason tripartite. ${ }^{25}$

Les écussons mamelouks sont regroupés en six formes, relevés et étudiés sur les monuments et les arts appliqués, le calice de l'échanson fait partie de chacune de ces six formes:

1. Un grand calice occupant la fasce et un autre, plus petit, en bas.

Cette forme était le plus souvent découverte sur les les monuments et les arts appliqués des émirs du sultan 'alZāhir Barqūq' et son fils 'al- Nāșir Farağ’, du 784 H./ 1382 J.C. jusqu’à 829 H./1426 J. C.

2. Sur une fasce, Un grand calice flanqué de deux autres calices, plus petits, et un petit calice en bas. Trouvé sur les monuments des émirs du sultan 'al- Mu'ayyad Šayh', entre 815 H./ 1412 J.C. et 871 H./ 1466 J. C. 
3. L'encrier du 'Dawādār' figurait au chef, deux calices sur la fasce et un losange à la base. Cette forme est apparue notamment sur les monuments des émirs du sultan 'al- Ašraf Barsbāy', entre 825 H./ 1422 J. C. et 863 H./ 1459 J. C.

4. Au chef, un losange, un grand calice sur la fasce et un autre, plus petit, à la base.

cette forme était probablement la base des écussons des mamalouks 'al- Z̄āhirriya Jaqmaq', ensuite, elle s'est étendue jusqu'au règne du sultan al- Ašraf Qāytbāy', entre 842 H./ 1338 J. C. et 900 H./ 1495 J. C.

5. L'encrier figurant au chef, un calice au milieu, et une fleur de lis en bas; ce blason appartenait à 'Ināl', voire même avant son sultanat, puis, il est devenu la forme originale d'où s'est émergé les blasons de ses Mameloukes al- Ašrafiyya Ināl.

6. Un losange au chef, sur la fasce, un calice surmonté d'un encrier et flanqué de deux cornes d'abondance, et un petit calice en bas. Cet écusson, déjà utilisé sous le règne de 'Jaqmaq', est devenu le blason de 'Qāytbāy' au moment de son sultanat; en 872 H./ 1468 J. C., puis, le blason de ses mamelouks et de ceux du sultan 'al- Ašraf Ğanblāṭ' (905 H./ 1500 J.C.). ${ }^{26}$

\section{Les tapis Mamelouks}

Le terme 'tapis', l'arabe 'țnfusa', vient du grec 'tapetion', probablement à travers l'araméen. ${ }^{27}$ Dans sa généralité, il désigne tout genre de revêtement textile. Le mode de vie et l'habitat, ainsi que les conditions climatiques, ont toujours engendré des attitudes variées à l'égard du tapis, celui- ci répondait à des fins pratiques, esthétiques et culturelles tout à la fois.

L'Egypte a connu le tissage des tapis depuis l'antiquité, il reste encore des traces de cet art conservé au musée Egyptien $^{28}$, une industrie qui continuait à exister pendant la période Gréco- Romaine ${ }^{29}$, il y a même des indices que l'Egypte ne se contentait pas de cette industrie locale, et qu'elle a eu recours à l'importation des produits tissés de la Syrie. ${ }^{30}$ Ensuite, l'Egypte byzantine a connu un épanouissement dans l'industrie du tissage, puisqu'on a des traces qui indiquent la profusion de ces produits et leurs divers usages. ${ }^{31}$ En effet, l'évolution de la tapisserie continuait sous les musulmans, c'est ainsi que les palais des califes abbassides ont été jonchés de navettes, tapis étendus sur sol et des tentures suspendues aux parois. ${ }^{32}$ Une tendance bien accentuée sous les fatimides qui tenaient beaucoup à la splendeur de leurs demeures et la magnificence de leur décor dont la tapisserie jouissait d'une extrême importance et de divers usages. ${ }^{33}$

Le tapis du sol, sous la forme du travail à points noués, a connu son plus grand essor et le summum de perfection artistique sous le règne des Mameloukes, fabriqués à partir du règne de Qāytbāy (r. 1469-1496) ${ }^{34}$, ils sont extrêmement rares et sont considérés comme les plus beaux tapis jamais produits.

Peut- être faut -il en outre supposer que les tapis Mameloukes faisaient partie des cadeaux offerts aux sultans, aux émirs et aux rois de différents pays comme le Yémen et le Maroc.

Les sources arabes nous fournissent tant d'exemples de l'échanges des tapis comme c'était le cas sous le règne du sultan 'Baybars' (660H./ 1261 J.C.), quand il a envoyé des cadeaux au roi de Constantinople, parmi lesquels plusieurs tapis. ${ }^{35}$

\section{Matériaux et techniques}

Principalement fabriqués de laine et de soie ${ }^{36}$ la tapisserie a pour origine une œuvre peinte ou dessinée: un modèle ou «petit patron», qui, agrandi aux dimensions de la tapisserie, devient un carton ou «grand patron»; et c'est alors qu'intervient le tisserand pour le traduire en tapisserie. De ce fait, la tapisserie est la transposition d'un modèle peint, certes, elle est étroitement liée à la peinture, mais sa fonction est différente, elle réchauffe l'atmosphère, absorbe la lumière, apaise les bruits, calme le regard et repose l'esprit.

Par la puissance de son décor, le tapis à points noués est un des emblèmes de l'art musulman, sa fabrication est simple, il comporte les éléments suivants:

- La chaine: La chaîne est formée des fils verticaux et parallèles, généralement de coton, tendus entre les deux extrémités du métier.

- Le noeud: il est formé en fixant un brin de laine sur deux fils de chaîne.

- La trame: La trame consiste en un ou plusieurs fils transversaux, presque toujours de coton, que l'on passe entre chaque rangée de nœuds. 
- $\quad$ Le velours: Le velours est la surface même du tapis : généralement en laine, il est formé de courts brins que l'on noue autour de la chaîne. Les nœuds s'alignent sur toute la largeur du tapis en cours d'exécution, mais jamais dans le sens de la hauteur.

- Les surjets ou lisières: ce sont des ourlets en laine de couleur placés tout au long des deux côtés du tapis pour renforcer celui-ci.

- Les franges: sont les extrémités visibles des fils de chaîne aux deux bouts du tapis.

Pour exécuter un tapis noué à la main, le tisserand utilisait le métier et des outils indispensables comme les ciseaux, couteaux, peigne et aiguille. ${ }^{37}$

L'essentiel de la technique du point noué consiste à confectionner un fond ou contexture, revêtu sur une face d'un velours constitué de courts brins de fil individuels. Il s'agit d'un travail purement manuel effectué sur un simple métier à tisser portant les fils de chaîne tendus parallèlement. Avec une alternance régulière se pratiquent le passage de plusieurs trames solidement attachées et l'exécution des différents rangs de nœuds ${ }^{38}$, ces derniers étant faits autour des fils de chaînes. ${ }^{39}$

Sa fabrication est simple. Après avoir tendu la chaîne, le tisserand introduit un bâton qui sépare celle-ci en deux nappes de fils. Une perche de lisses, fixée à l'extérieur du métier, amène sur le devant, au moyen de petites boucles (les lisses), tous les fils de la nappe arrière. Quand le bâton est abaissé sur la perche de lisses, il renvoie dans le fond tous ces fils. Quand on le relève, les fils arrière reprennent leur position. Ce système permet au tisserand de constituer avec sa trame une bande de tapisserie qui sera la lisière du tapis. Le nouage commence alors. A l'aide d'une lame à bout crocheté, le tisserand noue un brin de laine sur deux fils de chaîne consécutifs, procède de même sur les deux fils suivants et garnit ainsi toute une ligne. Sur celle-ci, la trame est généralement passée deux fois, puis serrée avec un peigne. Les boucles débordant au fond de la trame, étaient ensuite égalisées au ciseau et formaient une surface dense et élastique. ${ }^{40}$ Le nombre de nœuds dans des directions horizontales et verticales est identique, ce qui permet au tisserand de créer des cercles et des carrés parfaits et symétriques.

\section{Décor}

Les Musulmans exaltaient la fonction territoriale du tapis en l'associant au culte religieux, au protocole des cours, en encadrant son champ d'une sorte d'écriture. La conception du tapis mamelouk se caractérise souvent par un majestueux décor sur plan centré, au milieu du champ central, un grand octogone, généralement garnis d'un motif géométrique, et entouré de médaillons. Le champ central est grillagé d'éléments végétaux minuscules et stylisés tant que le papyrus et le cyprès; l'effet est celui d'un lanternon élevé sur assise carrée, tandis que la calligraphie arabe est parfois représentée sur les bordures ${ }^{41}$ Ces motifs sont créés dans une palette de trois couleurs des bijoux aux tons rouges, bleus et verts.

\section{Exemples de tapis portant le blason du calice}

\section{Tapis n ${ }^{\circ}$}

Fragment d'un tapis du règne du sultan Qāytbāy (r. 1468-1496), portant le blason d'échanson composé. ${ }^{42}$

Conservé au musée de textile à Washington D. C.

$\mathrm{N}^{\circ}$ d'inventaire: 1965.49 .1

Dimensions: $210 \times 220 \mathrm{~cm}$.

\section{Description}

Fragment d'un grand tapis du sultan Qāytbāy, de l'époque mamelouk circassienne, fabriqué en laine fine sur deux niveaux, la chaîne en laine ivoire et la trame en laine rouge foncé. (fig.1)

De plan rectangulaire, le champ central est octogonal à médaillon garnis de motifs géométriques et floraux, une admirable bordure principale répète des motifs floraux alignés, sur la partie inférieure de cette bordure, sont exécutés des ornements sous la forme de la coupe, formée d'un bourgeon d'où s'étendent deux feuilles latérales, constituant les bras de la coupe. ${ }^{43}$

La partie la plus remarquable de la bordure est et un carré comportant le blason de la coupe d'échanson. La bordure secondaire est grillagée de formes géométriques étoilées.

\section{Description du blason}

Exécuté dans un cadre carré de motifs floraux dorés et ondulés, le cadre renferme un octogone sur fond rouge foncé, à quatre écoinçons triangulaires garnis d'ornements floraux de couleurs ivoire, brun foncé et rose. 
Le blason est tripartite, dans un médaillon rayonnant en ivoire, la fasce comporte le calice d'échanson, dans lequel est exécuté l'encrier du dawādār, et flanqué de deux cornes d'abondance en vert jaunâtre, sur fond jaune moutard, au chef s'affiche le losange du Ğammdār sur fond rouge foncé, en bas, un petit calice de couleur ivoire sur fond brun foncé.

\section{Tapis n 2}

Fragment d'un tapis du règne du sultan Qāytbāy, comportant un blason d'échanson composé. ${ }^{44}$

Conservé au Museo Storico della Caccia e del Territorio, Florence, Italie.

$\mathrm{N}^{\circ}$ d'inventaire: Bd526_542

Dimensions: 456 x $945 \mathrm{~cm}$ (panneau A: $234 \mathrm{~cm}$ x 945; panneau B: 222 x $682 \mathrm{~cm}$ )

\section{Description}

Fabriqué en laine fine sur deux niveaux, la chaîne en laine ivoire et la trame en laine rouge foncé. (fig.2)

Tapis rectangulaire, de plan centré, autrefois, sous la forme d'un motif géométrique étoilé, renfermant des octogones grillagés d'ornements géométriques et floraux.

Le champ est exécuté sur fond rose pourpre, à deux médaillons centré en haut et en bas. Les écoinçons de la bordure principale sont ornés de quatre blasons d'échansons composés, (dont un est aujourd'hui perdu).

\section{Description des blasons}

Il parait que chaque deux blasons opposés sont identiques, ceux exécutés en haut sont encadrés de motifs floraux dorés et ondulés, le cadre renferme un octogone sur fond rouge foncé, à quatre écoinçons triangulaires garnis d'ornements floraux de couleurs ivoire et bleu azur, brun foncé et rose.

Le blason est tripartite, dans un médaillon en bleu azur, la fasce comporte le calice d'échanson, en bleu azur sur fond jaune moutard, dans lequel on voit l'encrier du dawādār, flanquée de deux cornes d'abondance noirs, au chef s'affiche le losange du Ğammdār, ivoire sur fond rouge foncé, en bas, un petit calice de couleur ivoire sur fond rose pourpre.

Quant aux blasons en bas du champ, toujours dans un octogone rouge foncé, tripartite dans un médaillon ivoire doré, la fasce comporte le calice avec l'encrier et les cornes, la différence dans celui-ci est qu'il est exécuté en vert, le losange en chef et un petit calice de couleur ivoire doré en bas.

\section{Tapis n $^{\circ} 3$}

Tapis Mamelouk, nommé Pulitzer, au nom de son acquisiteur, portant le blason d'échanson typique du règne du sultan Qāytbāy, toutefois, les couleurs sont différentes.

Conservé au Musée de Métropolitain, à New York. ${ }^{45}$

$\mathrm{N}^{\circ}$ d'acquisition: 1970.135

Dimensions: $421.6 \mathrm{~cm}$ x $345.4 \mathrm{~cm}$

\section{Description}

Tapis Mamelouke en bon état de conservation, fabriqué en laine sur deux niveaux, la chaîne en laine rouge foncée et la trame en laine brune. (fig.2)

De forme rectangulaire, toujours de plan centré, pourtant, cette pièce est caractérisée par la densité de ses ornements de motifs géométriques et floraux.

Un médaillon octogonal occupe le centre du champ, entouré de trois bordures garnis de divers motifs. Les écoinçons du champ sont ornés de quatre blasons d'échansons composés.

\section{Description des blasons}

Les blasons sont presque identiques, la seule différence réside dans la couleur du fond sur lequel il est exécuté. En haut du champ, les blasons apparaissent dans un rectangle ondulé en brun foncé ; dont les écoinçons sont garnis par des petits cercles ivoires, un octogone en rouge foncé entoure l'ovale du blason tripartite.

La fasce contient le calice de couleur noir sur fond jaune moutard, toujours avec l'encrier et les deux cornes, le losange au chef sur fond rouge foncé et un petit calice en bas sur fond vert noirâtre.

Ceux en bas représentent une forme identique sauf que la couleur du fond du chef est brune au lieu du rouge foncé. 


\section{Conclusion}

- Les armoiries étaient connues et portées par la noblesse sous les dynasties Ayyoubides, Mamelouks baharites et circassiennes.

- Quelques armoiries ont été accordées à titre temporaire. ${ }^{46}$

- Quelques armoiries, y compris le blason du calice, étaient héréditaires; les fils recevaient parfois le blason de leur père malgré qu'ils ne remplissent pas les fonctions liées à cette titulature.

- La plupart des tapis contenant des blasons mamelouks est conservée dans des musées à l'étranger, et dont la datation n'est pas précise.

- De formes simples ou composées, le blason de la coupe est apparu sur les tapis mamelouks.

- Les couleurs typiques qui caractérisaient les tapis Mameloukes sont le rouge, le bleu azur et le vert.

- Dans les pièces étudiées dans cette recherche, les différentes couleurs utilisées dans l'exécution du blason indiquent que ces pièces appartenaient à différents émirs puisque chaque échanson choisissait des couleurs spécifiques pour son blason à peur que ceux- ci soient mêlés.

- Il était difficile de déterminer le propriétaire de chaque pièce puisque les blasons étudiés sont presque identiques sauf en ce qui concerne les couleurs utilisées, ce qui indique qu'ils appartenaient au sultan 'Qāytbāy' ou un de ses mamelouks, au sultan 'al- Ašraf Ğanblāț' (905 H./ 1500 J.C.) et ses mamelouks, ou bien au sultan 'al- Ašraf Qanșuah al- Ġawrī'. ${ }^{47}$

\section{Annotations}

1. Artin, Y., Contribution à l'Etude du Blason en Orient, Londres, Picadilly, 1902, p. 1-2.

${ }^{2}$. Ibid., p. 4-5.

3. Ibid., p. 10-11.

Voir: Abū-al Qāsem al- Firdawsī (0940-1020), Le Livre des Rois, traduit par J. Mohel, Imprimerie Royale, Paris, (1838-1878).

${ }^{4}$. Le blason chez les Princes Musulmans 'Rank' est un mot qui a fait son introduction dans plusieurs langues européennes: «Un homme de rang est celui qui porte en écusson héraldique ou «rank».

- Rogers, E., Le Blason Chez les Princes Musulmans de l'Egypte et de la Syrie ; dans Bulletin de l'Institut Egyptien, deuxième série, $\mathrm{N}^{\circ} 1$, le Caire, 1880, p. 95.

5. Artin, Y., Contribution à l'Etude du Blason en Orient, p. 12.

6. 'Abdel Raziq, A., Al-Runūk al- Islāmiyya, Manšūrāt Kuliyat al- Ādāb gāmi 'at 'in Šams, Le Caire, 2000, p. 48.

7. Un terme composé de deux parties: Dawāh, un mot arabe qui signifie l'encrier et la plume, et dār, mot persan qui signifie "celui qui tient" ou "le responsable", alors, c'était une fonction remplie par celui qui est responsable des lettres sultaniennes et la signature du sultan, ainsi, il était toujours choisi des proches du souverain en qui il avait une confiance totale.

- al- Qalqašandī, (Abūl 'Abbās Aḥmad), Subḥ al- A ‘̌s̄ă, le Caire, 1914, t. 4, p. 19.

- al- Hुațīb, M., Mu 'gam al-Muștalaḥat wal- Alqāb al- Tārīhiyya, Mu'asasat al- Risāla, le Caire, 1996, p. 186.

${ }^{8}$. Un terme composé de deux parties: Silāh, un mot arabe qui signifie les armoiries, et dār, mot persan qui signifie "celui qui tient" ou "le responsable", alors, c'était une fonction remplie par celui qui est responsable des armoiries du sultan.

- al- Qalqašandī, Subḥ al- $A$ ‘̌s $\bar{a}$, t. 4, p 18, t. 5, p. 462.

9. Ahūr est un mot persan qui signifie la mangeoire des chevaux dans l'écurie, ainsi, émir ahūr était la titulature du responsable des écuries du sultan, il devait habiter à proximité des écuries, et être toujours prêt aux différentes transportations requises par le souverain.

- Ibid., t. 4, p. 18-19.

10. 'Abdel Raziq, A., al-Runūk al-Islāmiyya, p. 48.

${ }_{11}^{11}$ - Rogers, E., Le Blason Chez les Princes Musulmans, p. 110.

12. Yašbak min Mahdī était un émir qui a rempli les fonctions du Dawādār sous le règne du sultan Ğaqmaq et du sultan Khušqadam (871- 885 H./1466- 1480 J.C)

13 . Mayer, L. A., Saracenic Heraldy, Oxford, 1933, p. 252, n.3

${ }^{14}$. Daoud, M., al-Runūk al-Islāmiyya, al-Dāra, p. 29.

15. Muștafā, M., al-Runūk fì 'Așr al-Mamālīk, Magalat al- Risāla, N 400, 1941, p. 84.

${ }^{16}$. Un des mamluks d'al- Șāliḥ Nağm al- Dīn Ayyūb, devenu Sultan à l'époque des mamelouks baharites (620- 676H./ 1223 1277 J.C)

17. Fils du sultqn Qalāwūn, devenu sultan pendant trois périodes différentes: (693- 694 H./1293- 1294 J.C), (698- 708 H./ 1299 1309 J.C) et (709- 741 H./ 1309- 1341 J.C).

18. 'Abdel Raziq, A., al-Runūk al-Islämiyya, p. 159.

${ }^{19}$. Rogers, E., Les Blasons Chez les Princes Musulmans, p. 110.

20. 'Abd al- Nūr, H., al-Siğād al-Mamlūkī, Thèse de Doctorat, Faculté d'Archéologie, Université du Caire, 1991 , p. 394. 
${ }^{21}$. Décédé en 744 H./1343 J. C., et dont le blason est trouvé sur une lanterne de verre émaillé conservée au Musée d'Art Islamique au Caire.

22. Décédé en 824 H./1421 J.C., son blason de calice est gravé sur l'entrée Est de la mosquée Ommeyyade à Damas.

23. 'Abdel Raziq, A., Al-Runūk al-Islämiyya, p. 95.

${ }^{24}$. Ibid., p. 96.

25. Daoud, M., Al-Runūk al- Islāmiyya, p. 29.

${ }^{26}$. Ibid., p. $161-172$.

27. Wiet, G., Tapis Egyptiens, Arabica, T. 6, fasc.1, (Jan. 1959), p. 6.

28. Le Musée Egyptien conserve encore une navette bouclée qui a été découverte dans la tombe de Horemheb $\left(\mathrm{N}^{\circ} 31404\right)$, et d'autres textiles en lin remontant à la XIème dynastie $\left(\mathrm{N}^{\circ} 56279\right)$.

- Maher, S., al- Funūn al- Islāmiyya, al- Hay'a al- Misriyya l'l Kitāb, le Caire, 1986, p. 170.

${ }^{29}$. Marzūq, M., al- Funūn al- Zuhrufiyya al- Islamiyya fi Mișr qabl al- Fātimiyyīn, le Caire, 1974, p. 77.

${ }^{30}$. Khalīfa, M., Tārīh al- Mansūğgāt, Nahḍat Mișr, le Caire, 1961, p. 143.

31. Maher, S.; Gerges, H., Mansūgāt al-Mathaf al-Qibțì, le Caire, 1957, p. 48.

32. La majorité des tapis remontant à l'époque pré- fatimide est conservée au Musée de l'Art Islamique au Caire, tandis divers exemples, plutôt fabriqués en lin, en laine et portant des inscriptions et des dates de fabrication, sont exposés aux musée de textiles à Washington, le Métropolitain à New York, le musée National de Suède et bien d'autres musées.

32. 'Abd al- Nūr, H., Al-Sĭ̄ād al-Mamlūkī, p. 4.

33. Sous les musulmans, les tapis étendus sur le sol étaient liés au mode de vie puisque les gens n'utilisaient pas des salles à manger servies par des tables et des chaises, de sorte qu'ils mangeaient simplement par terre, sur le sol couvert de tapis tout en se servant de tables basses et de coussins.

- Ibid., p. 17.

34. Wiet a signalé dans son ouvrage «Tapis Egyptiens», que le palais de l'émir Quṣūn (m. 730 H./ 1330 J.C.) comportait des tapis d'Asie Mineure et de Diār Bakr, d'ailleurs, les seuls documents indiquant l'existence en Egypte de fabriques de tapis concernent les XVIème et XVIIème siècle.

- Wiet, G., Tapis Egyptiens, p. 4.

35. - Al- Maqrīzī, Taqī al- Dīn Aḥmad (d. 854 H.), al-Sulūk li Mi rifat Duwal al-Mulūk, vol. 1, p.2, p. $471-472$.

- Al- 'Aynī, Badr al- Dīn, 'Iqd al- Ğimān, p. 332., - Ibn Tag̉rī Bardī, al- Nuğūm al- Zāhira, t. 10, p 262., - Abū al- Fidā, alMuhtașar fì Ahbār al- Bašar, t. 4, p. 92-94.

36. Pour plus d'informations sur la fabrication de ces matières premières, voir: Māḍ̄i, I., Ziy Umarā' al- Mamālīk fi Miṣr wal' Šàm, le Caire, 2009, p. 35-39.

37. Fu'ād, A.; Muhammad, M., Fan al- Siğād al- Yadawī, Dār al- Ma'ārif, le Caire, 1963, p. 7-8.

${ }^{38}$. Le style des nœuds utilisé dans presque tous les tapis Mameloukes, est connu sous le nom 'Siné', qui était un petit village à Kurdistan.

- 'Abd al- Nūr, H., Al- Siğād al-Mamlūkī, p. 293.

39. Heinz, D., Tapis, Encyclopadia Universalis [en ligne].

${ }^{40}$. Migeon, G., Les Arts du Tissu, Paris, 1909, p 384.

41. En 1905, la découverte de huit tapis seljukides, dans la mosquée de 'Alā' al- Dīn de Konya auxquels s'ajoutent trois pièces un peu plus tardives trouvées dans la mosquée de Beyshehir, contribuait à éclaircir la conception du décor de la tapisserie Islamique.

${ }^{42}$.http://www.azerbaijanrugs.com/mamluk/sultan_qaitbay_mamluk_carpet_textile_museum_washington.htm.

${ }^{43}$. Ce motif floral décoratif était très répandu sur les tapis Mameloukes; peut-on supposer une relation avec le poste d'échanson à l'époque, surtout qu'il parait parfois seul et d'autres fois sur une même pièce comportant un blason d'échanson.

- Tawfīq, M., Istih̆dām al- Qiyam al- Gamaliyya wal' Asālīb al-tațbīqiyya fi fan al-Siğād al - Mașrī al-Ma 'qūd, Thèse de magistère, Faculté des Arts Appliqués, Université du Caire, 1974, p. 238.

${ }^{44}$.http://www.azerbaijanrugs.com/mamluk/sultan_qaitbay_mamluk_carpet_museo_storico_della_caccia_bardini.htm.

${ }^{45}$.http://www.azerbaijanrugs.com/mamluk/Pulitzer_Mamluk_carpet_the_metropolitan_museum_of_art.htm

${ }^{46}$. Rogers, E., Les Blasons Chez les Princes Musulmans, p. 101.

47. 'Abdel Raziq, A., Al-Runūk al- Islāmiyya, p. 161- 172.

\section{رنك الكأس على السجاد المملوكي}

ظهرت الرنوك بكثرة على التحف والآثار التي صنعت برسم سلاطين وأمراء الدولة المملوكية بشقيها البحري والجركسي، وقد تتوعت بين الرنوك

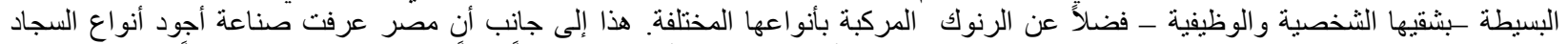

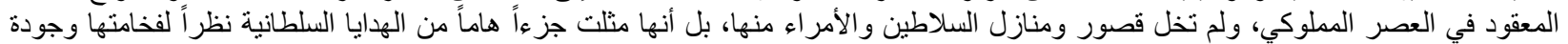

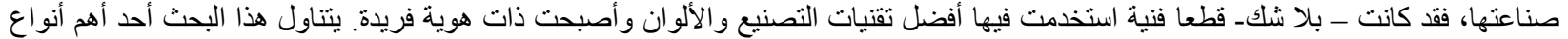

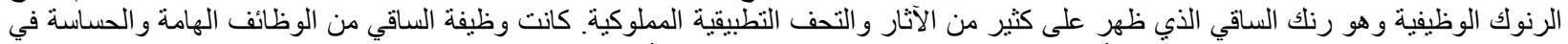

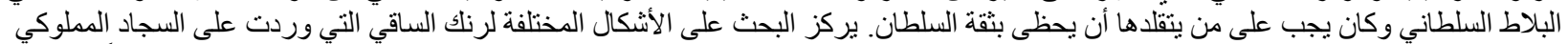

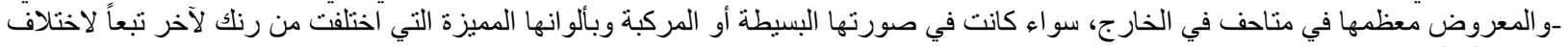
صاحب الرنك.

$$
\text { الكلمات الدالة: الرنوك- رنك الكأس ـ السجاد المملوكيـ الفنون التطبيقية. }
$$




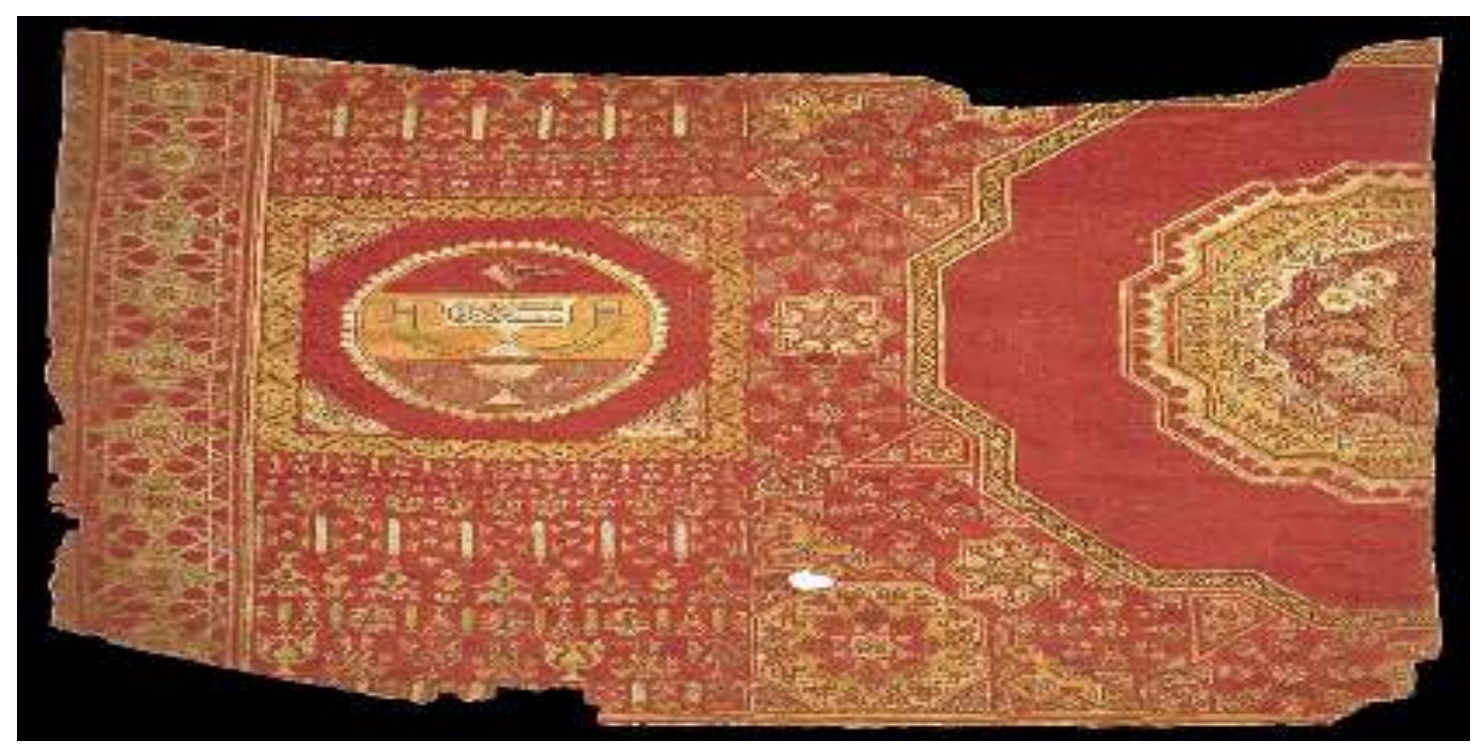

Fig. 1- Tapis portant le blason du calice, règne du sultan Qāytbāy (1468-1496 J.C.)

Musée de Textile, Washington D. C, n 1965.49 .1

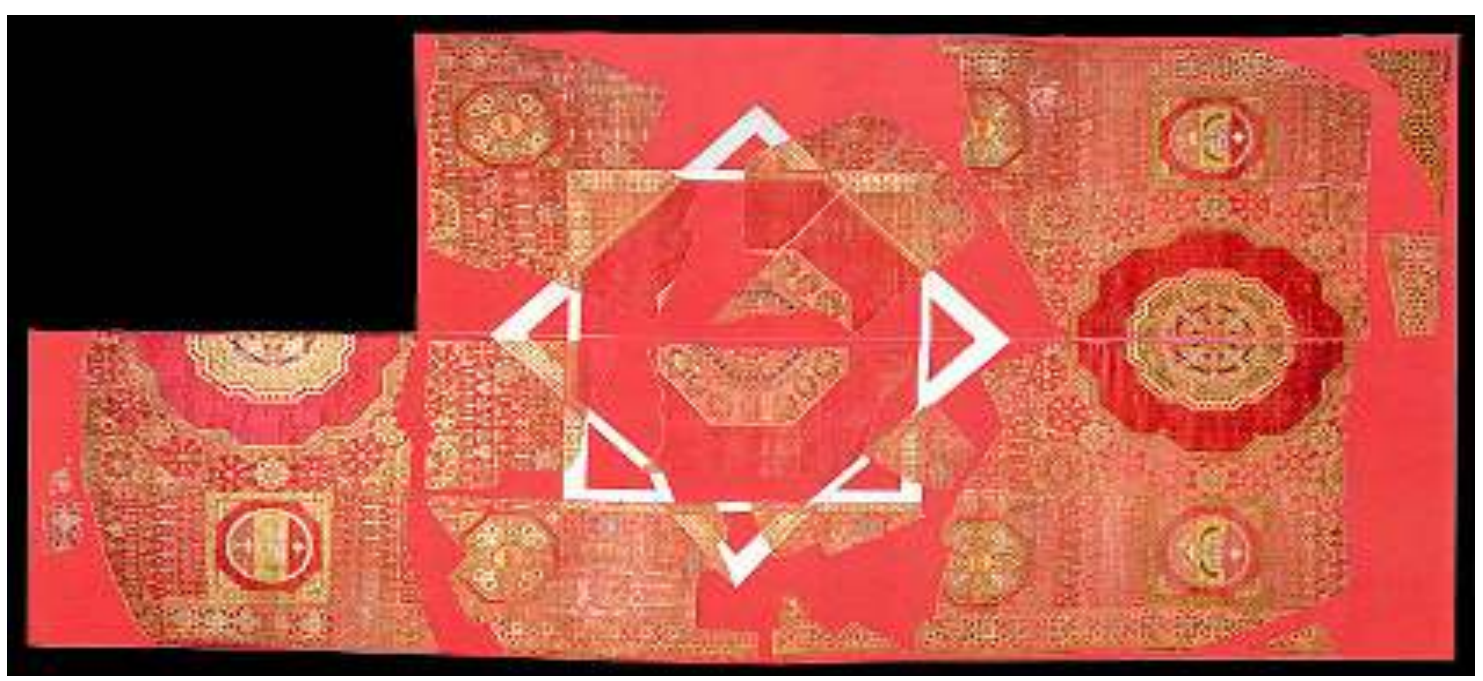

Fig. 2- Fragment du tapis portant le blason du calice, règne du sultan Qāytbāy (1468-1496 J.C.)

Museo Storico della Caccia e del Territorio, Florence, Italie, n ${ }^{\circ}$ Bd526_542 


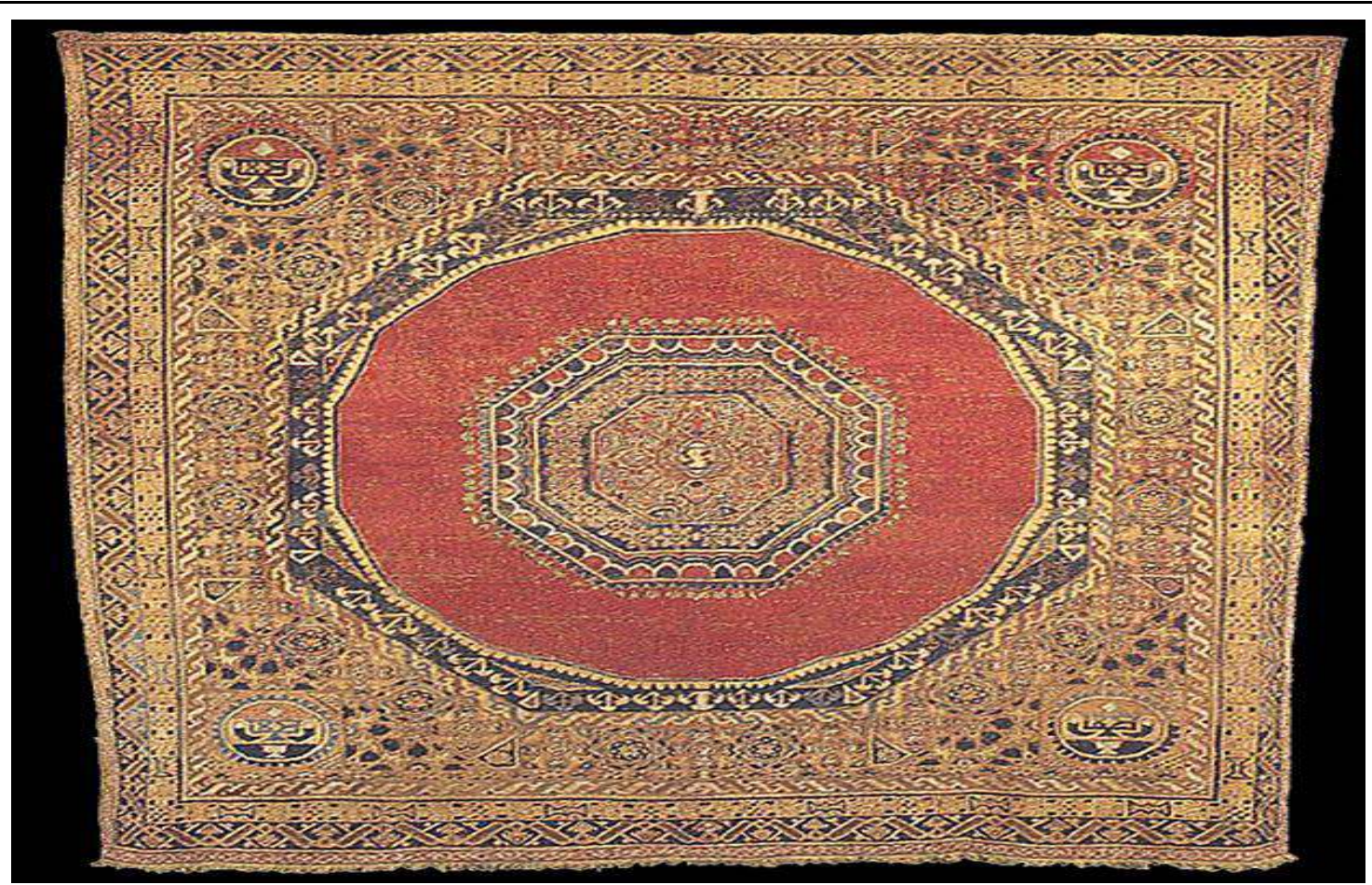

Fig. 3-a- Tapis Mamelouke comportant le blason d'échanson

Metropolitan Museum of Art, New York

(Acheté par Joseph Pulitzer Bequest, 1970), nº d'acquisition: 1970.135

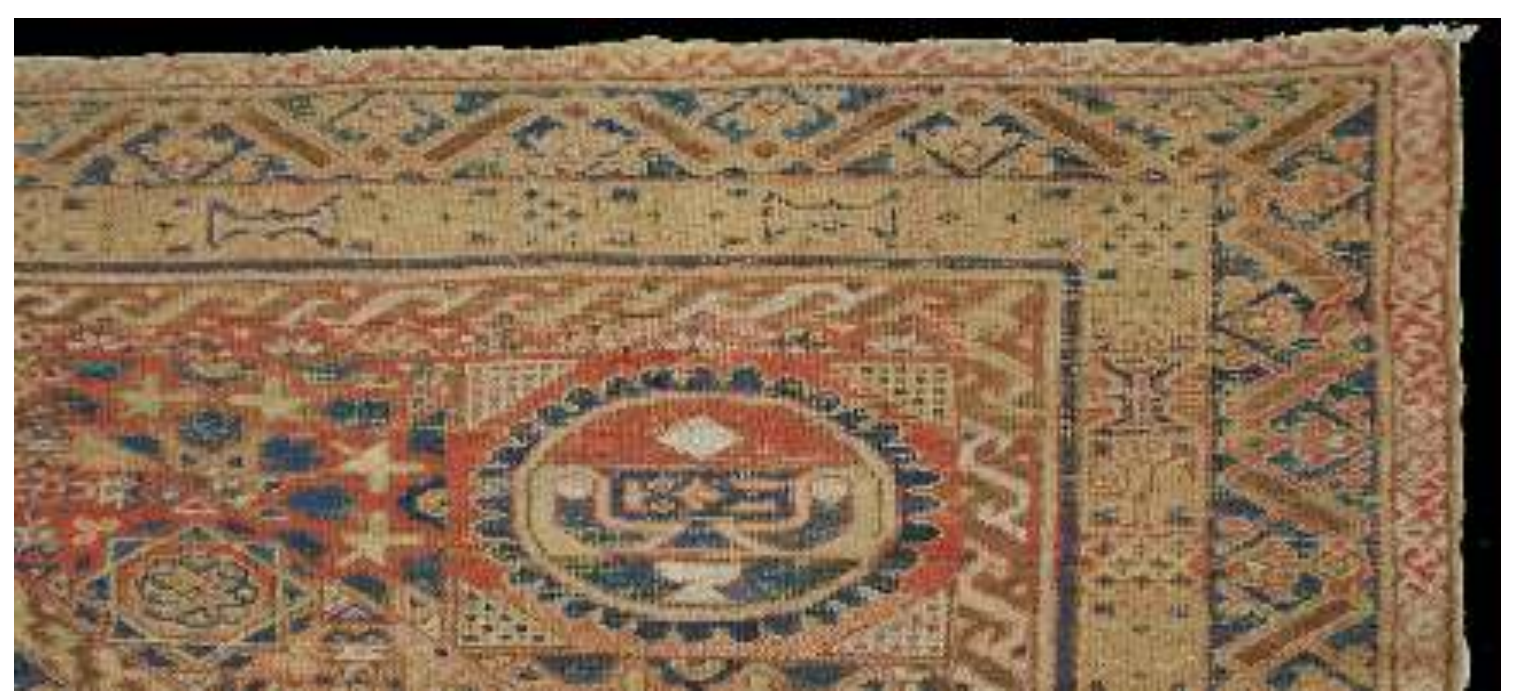

Fig. 3-b - Détails du blason d'échanson

Metropolitan Museum of Art, New York

(Acheté par Joseph Pulitzer Bequest, 1970), n d'acquisition: 1970.135 